\title{
Missed Opportunities: Optimal Investment Timing when Information is Costly*
}

\author{
Graeme Guthrie ${ }^{\dagger}$ \\ Victoria University of Wellington
}

August 3, 2005

${ }^{*}$ The author gratefully acknowledges the helpful comments of Glenn Boyle.

${ }^{\dagger}$ Address: School of Economics and Finance, PO Box 600, Victoria University of Wellington, Wellington, New Zealand. Ph: 64-4-4635763. Fax: 64-4-4635014. Email: graeme.guthrie@vuw.ac.nz 


\title{
Missed Opportunities: Optimal Investment Timing when Information is Costly
}

\begin{abstract}
Real options analysis typically assumes that projects are continuously evaluated and launched at precisely the time determined to be optimal. However, real world projects cannot be managed in this way because of the costs of formally evaluating an investment opportunity. This paper analyzes how projects should be managed in such a world. Information about a project comes in two flavors: project-specific information, which can only be observed if a fixed evaluation cost is incurred; and generic information, which can be observed without cost. If sufficiently good generic information is observed during the period immediately after a project evaluation, the firm will invest without any further evaluation. Beyond this period, the firm will always reevaluate the project before investment. The availability of information and project evaluation costs affect the firm's optimal behavior in different ways: a higher evaluation cost means that the firm evaluates the project later (that is, sets a higher evaluation threshold) but invests sooner (that is, sets a lower investment threshold); a bigger role for project-specific information also induces the firm to set a lower investment threshold, but it actually encourages the firm to evaluate the project sooner. The value of waiting is lower when more information is project-specific and when project evaluations are more costly. Standard real option models may therefore overestimate the value of investment timing flexibility. This misvaluation is especially severe when the value of the completed project is strongly mean reverting, because then precision in investment timing is particularly important.
\end{abstract}

JEL Classification code: G31, D81

Keywords: real options, incomplete information, capital budgeting 


\section{Missed Opportunities: Optimal Investment Timing when Information is Costly}

\section{Introduction}

Real options analysis uses techniques developed for pricing financial derivatives to analyze real investment projects. While this approach has created significant insights into optimal investment policies, there are fundamental differences between financial derivatives and real investment projects. Consider the example of an American call option on a dividend-paying stock. It is possible to calculate the optimal exercise policy in terms of a stock price threshold above which immediate exercise is optimal. The same approach has been applied to the option to invest in a real investment project, allowing the optimal investment timing policy to be derived. However, while it is (almost) feasible for the owner of the call option to continuously monitor the share price, and thereby exercise the option at exactly the right time, it is not feasible to continuously re-evaluate the payoff from a complicated real investment project. While some information (such as commodity prices, exchange rates, and interest rates) can be observed almost costlessly and, for many projects, its impact on the value of a project easily calculated, such information will not generally allow an exact calculation of project value to be made. For this, further, perhaps project-specific, information must be gathered and this process of project evaluation is more costly. The cost of evaluating this payoff renders continuous evaluation impractical, but this limitation is not incorporated into existing real option models. This paper therefore extends a standard real option model (see, for example, McDonald and Siegel (1986)) by distinguishing these two sources of information and analyzing the effect of the amount of project-specific information, and the cost of gathering and processing it, on firms' optimal investment policies.

In the first part of this paper I present a model in which the only source of information about an investment project is project-specific. A firm, which has the perpetual right to invest in the project, can only observe this information if it incurs some fixed evaluation $\operatorname{cost}^{1}$; the firm does not receive any information about the project's value between such evaluations. The firm's optimal policy is to decide at the time of each evaluation how long to wait before reevaluating the project. Immediately after an evaluation, the firm will invest if the project's net present value (NPV) exceeds a strictly positive threshold that reflects the value of the investment timing option which is lost when the firm invests; it will abandon the project if the NPV lies below a strictly negative threshold; otherwise it will wait a fixed period of time before reevaluating the project. I find that the firm will wait a short time before reevaluating projects that currently

\footnotetext{
${ }^{1}$ This cost can be thought of as a "deliberation cost" of the type considered in the literature on bounded rationality (Conlisk, 1996).
} 
have a high NPV, and a long time before reevaluating projects that currently have a low NPV.

When the cost of evaluating the project rises, the firm waits longer before reevaluating the project and sets its investment and abandonment thresholds closer to the break even point. In the limit as project evaluations become infinitely costly, the optimal investment rule approaches the standard NPV rule. A higher evaluation cost lowers the value of the firm's investment timing option in part because of the cash outflows which result from evaluating the project. However, evaluation costs are also 'costly' because the resulting low frequency of project evaluations means that the firm cannot time its investment with sufficient precision. The value of the investment rights comes from having the flexibility to invest at exactly the right time, but if the project is evaluated infrequently then a valuable opportunity to invest might be missed. This is less important when project value follows a geometric Brownian motion, since stochastic shocks to project value are permanent in this case, but more important when project value is mean reverting, since the cost of missing the 'peak' project value can be significant. In fact, even small evaluation costs can dramatically lower the value of waiting in this case. ${ }^{2}$

In the second part of the paper I extend this model by supposing that some information, which I call generic information, about the project's value is costlessly available and that the firm can only observe the remaining (project-specific) information after incurring an evaluation cost. I find that if generic information provides sufficiently good news during the period immediately after a project evaluation, the firm will simply invest without any further evaluation. However, beyond this period, the firm will always reevaluate the project before investment. Such evaluations will occur as soon as the project's estimated value (based on generic information) exceeds some threshold. As the time since the last evaluation grows, the standard required for reevaluation falls; if a sufficiently long time has elapsed, the firm will even reevaluate a project which it expects has a negative NPV.

As might be expected, the project rights are less valuable when more information is projectspecific and when project evaluations are more costly. However, the availability of information and project evaluation costs have subtly different effects on the firm's optimal behavior: a higher evaluation cost means that the firm evaluates the project later (that is, sets a higher evaluation threshold) but invests sooner (that is, sets a lower investment threshold); a greater amount of project-specific information also induces the firm to invest sooner, but it leads the firm to evaluate the project sooner (not later).

\footnotetext{
${ }^{2}$ Schwartz (1997) shows that firms generally set their investment threshold too high if they ignore mean reversion. However, Metcalf and Hasset (1995) argue that the predicted timing of investment may not be significantly altered because the greater volatility that results from ignoring mean reversion means that any threshold will be reached earlier. This finding has recently been challenged by Sarkar (2003). The results of the current paper suggest that the presence of mean reversion can dramatically alter optimal investment, in a way not considered by these authors, when projects are costly to evaluate.
} 
These different effects can be understood in terms of the firm's two timing options. Firstly, the firm must decide when to evaluate the project, which it will only do once the payoff from project evaluation exceeds the value of waiting. A higher evaluation cost lowers the payoff, and so delays evaluation. If there is a great deal of project-specific information, then delaying evaluating the project yields a relatively small amount of generic information; thus, the value of waiting is low, and, all else equal, evaluation occurs sooner. Secondly, the firm must decide whether to launch the project or wait and reevaluate it in the future; it will only launch the project if the payoff from doing so exceeds the value of waiting. A higher evaluation cost lowers the value of waiting (by making future evaluations more expensive), and therefore promotes immediate investment. More project-specific information means that delaying yields a relatively small amount of generic information, lowering the value of waiting, and therefore also promoting immediate investment.

A number of recent papers also look at the problem of pricing options with incomplete information. Merton (1998) values options when the price of the underlying asset can only be observed at some initial date and then immediately after the option is exercised, but the prices of other assets (which are correlated with the unobservable variable) can be observed. I use his valuation framework. Childs et al. (2001) value options when the underlying asset's value is observed with noise. They consider the optimal exercise policy for a McDonald-Siegel style investment timing option, but in their example the firm does not have the option to acquire information on the project's true value. ${ }^{3}$ Epstein et al. (1999) consider a firm with a perpetual option to invest in a project of unknown value when the firm is able to observe a noisy signal of this value while it waits to invest, but in their model the value of the underlying asset is constant. Martzoukos and Trigeorgis (2001) model the option to undertake an investment at some fixed future date when information about the final payoff can be acquired at specified dates prior to the option's expiry date. However, the timing of information acquisition and investment is exogenous (in particular, the investment options are European in nature), so that the effect of evaluation costs on the timing of the firm's actions cannot be examined.

Suggested capital budgeting rules range from the standard NPV analysis, in which projects are assumed to be of the now-or-never variety, to real options analysis, in which projects often have maximal flexibility. Recently some authors have followed a path between these two extremes, recognizing that firms do not necessarily have the degree of flexibility often assumed by popular real option models. For example, it has been argued that competition reduces the value of investment timing options (Grenadier, 2002; Lambrecht and Perraudin, 2003), although the generality of this result has recently been questioned (Novy-Marx, 2004). Others

\footnotetext{
${ }^{3}$ Childs et al. do study costly information-acquisition, but only in the context of secured debt, and then information is only acquired at some fixed date. Thus they cannot say much about when the firm should acquire information.
} 
have argued that external financing constraints (Boyle and Guthrie, 2003, 2005) and bondholder/shareholder conflict (Lyandres and Zhdanov, 2003; Mauer and Sarkar, forthcoming; Sabarwal, 2003) can lower the value of waiting. Hugonnier and Morellec (2004) examine the impact of manager/shareholder conflict on investment timing and find that it lowers the value of waiting, while Grenadier and Wang (2005) argue that managers will tend to invest later than is optimal for the firm's owners. The current paper contributes to this literature by showing that something as simple as a small cost incurred whenever the project is evaluated for investment can also significantly reduce the value of a firm's option to delay investment.

I present the basic model, which modifies a simple model of investment timing by assuming that only project-specific information is available, in the next section. Generic information is added to the basic model in Section 3, and my conclusions are summarized in Section 4.

\section{Optimal investment timing when information is costly}

The model is described in Section 2.1 in terms of a general diffusion process. Section 2.2 makes the common assumption that project value evolves according to geometric Brownian motion, while a popular mean reverting process is considered in Section 2.3.

\section{$2.1 \quad$ Model set-up}

I use a variant of the model of investment timing in McDonald and Siegel (1986) in which a firm has the perpetual right to invest in a project. Investment is irreversible and can occur at most once. The project costs $I$ to build and the completed project is worth $V_{t}$ at date $t$. I suppose that the firm cannot observe $V_{t}$ prior to investment unless it undertakes a formal project evaluation, which $\operatorname{costs} C$ and immediately reveals the current value of $V_{t}$. Furthermore, I suppose that the firm cannot infer anything about $V_{t}$ from publicly available information. In particular, I assume that changes in $V_{t}$ are uncorrelated with changes in the prices of traded securities. ${ }^{4}$ The risk from holding the completed project is therefore diversifiable, so I assume that the expected rate of return from holding the completed project is equal to the riskless interest rate $r$. That is, $V_{t}$ evolves according to

$$
d V_{t}=\left(r V_{t}-\delta\left(V_{t}\right)\right) d t+\sigma\left(V_{t}\right) d \xi_{t}
$$

where $\xi_{t}$ is a Wiener process and $\delta\left(V_{t}\right)$ is the cash flow generated by the completed project at date $t$.

Since no other source of information about the project's value is available to the firm between project evaluations, the value of the project rights at date $t$ depends on the time elapsed since

\footnotetext{
${ }^{4}$ In Section 3 I analyze the situation where movements in the prices of traded securities reveal information about changes in the value of the completed project.
} 
the project was last evaluated and the value of the (hypothetical) completed project at that date; that is, the value of the project rights at date $t$ equals $F\left(V_{T}, t-T\right)$ for some function $F$, where $T$ is the date of the most recent evaluation.

At each date $t$ prior to investment, the firm must do one of the following: (i) abandon the project rights altogether, and receive a payoff of 0; (ii) invest immediately, and receive an expected payoff of $E_{t}\left[V_{t}\right]-I$; (iii) reevaluate the project, and receive an expected payoff of $E_{t}\left[F\left(V_{t}, 0\right)\right]-C$; or (iv) do nothing for the next interval of length $d t$, and receive a payoff of $e^{-r d t} F\left(V_{T}, t+d t-T\right) .{ }^{5}$ Thus, the value of the project rights at date $t$ is

$$
F\left(V_{T}, t-T\right)=\max \left\{0, E_{t}\left[V_{t}\right]-I, E_{t}\left[F\left(V_{t}, 0\right)\right]-C, e^{-r d t} F\left(V_{T}, t+d t-T\right)\right\} .
$$

Notice that no new information about the project's viability is available between formal evaluations. Therefore, if the firm decides to neither abandon the project, nor invest, immediately after a project evaluation, then it can optimally choose the timing of its next action there is no point in delaying deciding when to act next. This allows me to concentrate on the firm's decision immediately after it evaluates the project. The optimal policy immediately after a project evaluation is to (i) abandon the project altogether, (ii) commit to invest $\tau$ years in the future (for some $\tau \geq 0$ ), or (iii) commit to reevaluate the project $\tau$ years in the future (for some $\tau>0)$. The respective payoffs are 0 ,

$$
e^{-r \tau}\left(E_{t}\left[V_{t+\tau}\right]-I\right)
$$

and

$$
e^{-r \tau}\left(E_{t}\left[F\left(V_{t+\tau}, 0\right)\right]-C\right)
$$

where $t$ is the current date. ${ }^{6}$ Thus the project rights are worth

$$
F\left(V_{t}, 0\right)=\max \left\{0, \sup _{\tau \geq 0} e^{-r \tau}\left(E_{t}\left[V_{t+\tau}\right]-I\right), \sup _{\tau>0} e^{-r \tau}\left(E_{t}\left[F\left(V_{t+\tau}, 0\right)\right]-C\right)\right\}
$$

immediately after a project evaluation.

The firm's optimal investment policy can be found by solving equation (1) for $F$. The complexity of this problem demands that solutions are found using numerical techniques, which are described in Appendix A. I find thresholds $\underline{V}$ and $\bar{V}$ such that the firm should abandon the project whenever an evaluation reveals that $V_{t} \leq \underline{V}$, commit to investing in the project in

$$
\tau^{i}\left(V_{t}\right)=\arg \max _{\tau \geq 0} e^{-r \tau}\left(E_{t}\left[V_{t+\tau}\right]-I\right)
$$

\footnotetext{
${ }^{5}$ In case (iv), the future payoff of $F\left(V_{T}, t+d t-T\right)$ is riskless, explaining why it is discounted at the riskless interest rate.

${ }^{6}$ The expected payoffs from future investment, $E_{t}\left[V_{t+\tau}\right]-I$, and evaluation, $E_{t}\left[F\left(V_{t+\tau}, 0\right)\right]-C$, are discounted at the riskless interest rate due to the assumption that shocks to $V_{t}$ are uncorrelated with changes in the prices of traded securities, which implies that $V$-risk is diversifiable.
} 
years if $V_{t} \geq \bar{V}$, and otherwise wait

$$
\tau^{e}\left(V_{t}\right)=\arg \max _{\tau>0} e^{-r \tau}\left(E_{t}\left[F\left(V_{t+\tau}, 0\right)\right]-C\right)
$$

years before reevaluating the project. The cost associated with a high value of $\tau^{e}$ is that receipt of the eventual payoff is delayed. However, waiting a long time before reevaluating the project allows greater variability in $V_{t+\tau}$ and, provided $F(V, 0)$ is convex in $V$, a greater expected payoff. A positive trend in $V_{t+\tau}$ adds to this benefit. The optimal timing of project evaluations equates the marginal cost of delaying the next evaluation and the marginal benefit.

\subsection{Geometric Brownian motion}

In this section I suppose that the completed project generates cash flow of $\delta V_{t}$ at date $t$, for some constant $\delta$, and that $\sigma\left(V_{t}\right)=\sigma V_{t}$ for some constant $\sigma$. This implies that $V_{t}$ evolves according to the geometric Brownian motion

$$
d V_{t}=(r-\delta) V_{t} d t+\sigma V_{t} d \xi_{t}
$$

I assume that $\delta>0$, which ensures that there is an opportunity cost of delaying investment. ${ }^{7}$

Figure 1 plots the value $F(V, 0)$ of the project rights as a function of the value $V$ of the completed project, for the situation where $r=0.04, \delta=0.04, \sigma=0.2$, and $I=1$. The solid curve corresponds to the case where the evaluation cost is $C=0.001$, or 0.1 percent of the project's construction cost. The dashed and dotted curves plot the same function, but for $C=0.01$ and $C=0.05$ respectively. It is optimal to invest immediately when $F(V, 0)=V-I$, and to abandon the project altogether when $F(V, 0)=0$. Otherwise, the project should be reevaluated at some future date. The figure reveals that although the presence of evaluation costs does not eliminate the value of waiting, it does lower the value of the firm's investment timing option.

Evaluation costs lower the value of the project rights for two reasons. First, the firm incurs cash outflows each time it evaluates the project. Second, the firm's investment timing is distorted away from that which is optimal in the absence of evaluation costs. I write $F(V, 0)=P_{C}^{i}(V)-$ $P^{e}(V)$, where $P_{C}^{i}(V)$ equals the present value of the project's ultimate payoff (that is, either $V_{t}-I$ or zero) and $P^{e}(V)$ equals the present value of the series of evaluation costs, both calculated assuming that the firm follows the investment and evaluation policy that maximizes $F(V, 0)$. If $P_{0}^{i}(V)$ equals the value of the project rights when $C=0$, then the loss in value equals

$$
P_{0}^{i}(V)-F(V, 0)=\left(P_{0}^{i}(V)-P_{C}^{i}(V)\right)+P^{e}(V)
$$

\footnotetext{
${ }^{7}$ In the standard case where the project's evaluation cost is zero, the firm would never invest if $\delta \leq 0$; assuming $\delta>0$ ensures that there is a finite investment threshold in this case.
} 
Figure 1: Value of the project rights

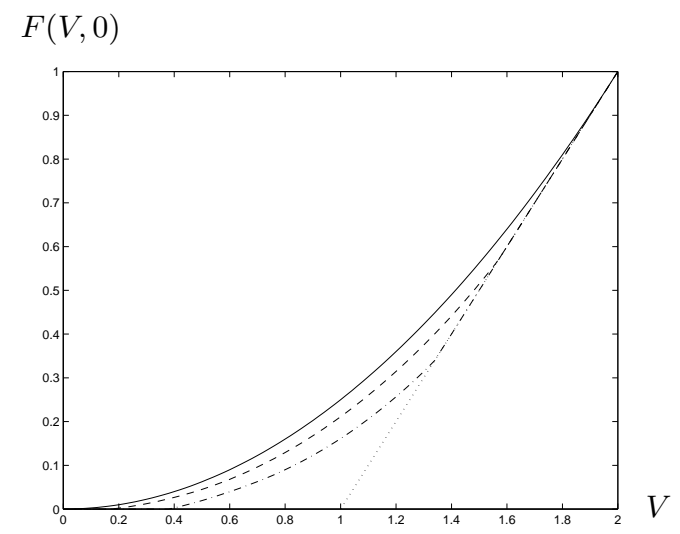

Notes. The solid curve plots the value $F(V, 0)$ of the project rights as a function of the value $V$ of the completed project, in the special case where $C=0.001$. The dashed and dotted curves plot the same function, but for $C=0.01$ and $C=0.05$ respectively. It is optimal to invest immediately when $F(V, 0)=V-I$, and to abandon the project altogether when $F(V, 0)=0$. Otherwise, the project should be reevaluated at some future date. Project value evolves according to the geometric Brownian motion process in (2). In all cases, $r=0.04, \delta=0.04, \sigma=0.2$, and $I=1$.

The expression inside the brackets on the right side, $P_{0}^{i}(V)-P_{C}^{i}(V)$, can be interpreted as the cost of the distorted investment timing, because $P_{0}^{i}(V)$ and $P_{C}^{i}(V)$ each equal the present value of the project's ultimate payoff, but calculated using different investment policies, $P_{0}^{i}(V)$ for when evaluations are costless and $P_{C}^{i}(V)$ for when they are costly. Meanwhile $P^{e}(V)$ equals the cost of carrying out the required evaluations.

Table 1 reports evidence on the magnitude of the loss in value of the project rights, and also on the relative importance of the two contributors to the loss in value. The first row of the table gives the loss in value of the project rights $\left(P_{0}^{i}(V)-F(V)\right)$, expressed as a percentage of $P_{0}^{i}(V)$. The second row gives the loss in value that is due to the altered investment timing $\left(P_{0}^{i}(V)-P_{C}^{i}(V)\right)$, expressed as a percentage of $P_{0}^{i}(V)$. The third row gives the loss in value that is due to the cost of carrying out the required evaluations $\left(P^{e}(V)\right)$, expressed as a percentage of $P_{0}^{i}(V)$. For example, if the cost of evaluating the project is one percent of its cost of construction and $V=I$, then the value of the project rights is 15.4 percent lower than if evaluation was costless. The present value of the actual expenditure on evaluations comprises 8.0 percent of the benchmark value, while the induced distortion of investment timing comprises 7.4 percent of the benchmark value. Inspection of the table shows that even small evaluation costs can significantly reduce the value of the project rights. Furthermore, the expenditure on project evaluation makes up just part of the loss in value. A second, indirect, effect - the consequences for investment timing - is of roughly the same order of magnitude. This indirect effect is discussed now. 
Table 1: The effect on the value of the project rights

\begin{tabular}{lccccccccc}
\hline \hline & \multicolumn{3}{c}{$C=0.01$} & \multicolumn{3}{c}{$C=0.02$} & \multicolumn{3}{c}{$C=0.05$} \\
& $V=0.8$ & $V=1.0$ & $V=1.2$ & $V=0.8$ & $V=1.0$ & $V=1.2$ & $V=0.8$ & $V=1.0$ & $V=1.2$ \\
\hline \% of value lost & 18.9 & 15.4 & 12.5 & 26.1 & 21.0 & 16.9 & 43.2 & 35.1 & 28.4 \\
. f from distorted timing & 9.4 & 7.4 & 5.9 & 14.7 & 11.8 & 9.5 & 23.5 & 20.0 & 16.8 \\
.. from evaluation costs & 9.5 & 8.0 & 6.6 & 11.4 & 9.2 & 7.4 & 19.7 & 15.0 & 11.6 \\
\hline \hline
\end{tabular}

Notes. The first row of the table gives the loss in value of the project rights $\left(P_{0}^{i}(V)-F(V)\right)$, expressed as a percentage of $P_{0}^{i}(V)$. The second row gives the loss in value that is due to the altered investment timing $\left(P_{0}^{i}(V)-P_{C}^{i}(V)\right)$, expressed as a percentage of $P_{0}^{i}(V)$. The third row gives the loss in value that is due to the cost of carrying out the required evaluations $\left(P^{e}(V)\right)$, expressed as a percentage of $P_{0}^{i}(V)$. The baseline parameter values are $r=0.04, \delta=0.04, \sigma=0.2$, and $I=1$.

Table 2 illustrates the effect of the evaluation cost on the firm's optimal investment policy. The first row of the top panel gives the optimal investment threshold for different levels of the evaluation cost, while the first row in the bottom panel gives the optimal abandonment threshold. For example, when the cost of evaluating the project is one percent of its construction cost, it is optimal to invest immediately after a project evaluation if the value of the completed project would be greater than 1.58, abandon the project rights altogether if the completed project would be worth less than 0.13 , and delay for intermediate values. Each cell in the bottom two rows of each panel shows the percentage change in the corresponding threshold resulting from a one percent change in the corresponding parameter.

Higher evaluation costs make it more expensive to delay the investment decision until more information is available, since this information is more costly to evaluate. Thus, they induce the firm to set a lower investment threshold and a higher abandonment threshold. As the evaluation cost rises, the optimal investment rule approaches the standard NPV rule; that is, the firm invests if the project's NPV is positive, and otherwise rejects the project. Note that even though the firm has perpetual rights to the project, as evaluation costs grow higher, the firm chooses to make the project a 'now-or-never' proposition.

When $\delta$ increases, the opportunity cost of delaying investment (that is, the foregone project cash flow) increases, and the expected growth rate in the value of the completed project falls. Both factors encourage the firm to invest earlier; that is, $\bar{V}$ falls. The second factor also makes it more difficult for the firm to justify incurring the costs required for future evaluations, so that the firm is also encouraged to abandon poor projects; that is, $\underline{V}$ rises. A similar story holds for the volatility of the completed project's value. A higher value of $\sigma$ raises the value of the firm's delay option. This raises the optimal investment threshold and, because it is easier to justify incurring future evaluation costs, lowers the abandonment threshold. These comparative static 
Table 2: Optimal investment policy

\begin{tabular}{|c|c|c|c|c|}
\hline & $C=0.01$ & $C=0.02$ & $C=0.05$ & $C=0.10$ \\
\hline \multicolumn{5}{|c|}{ Investment threshold } \\
\hline $\bar{V}$ & 1.58 & 1.50 & 1.35 & 1.22 \\
\hline$\delta$ & -0.57 & -0.56 & -0.55 & -0.49 \\
\hline$\sigma$ & 0.51 & 0.47 & 0.41 & 0.33 \\
\hline \multicolumn{5}{|c|}{ Abandonment threshold } \\
\hline$\underline{V}$ & 0.13 & 0.18 & 0.30 & 0.44 \\
\hline$\delta$ & 2.00 & 1.89 & 1.48 & 1.46 \\
\hline$\sigma$ & -2.00 & -1.58 & -1.29 & -1.01 \\
\hline
\end{tabular}

Notes. The top panel illustrates the sensitivity of the threshold, $\bar{V}$, that determines when the firm should launch the project to changes in parameters describing the stochastic structure of the completed project's value. The first row gives the optimal investment threshold for different levels of the evaluation cost. Each cell in the following two rows shows the percentage change in the investment threshold resulting from a one percent change in the corresponding parameter. The bottom panel repeats the analysis for the threshold, $\underline{V}$, that determines when the firm should abandon the project rights. Project value evolves according to the geometric Brownian motion process in (2). The baseline parameter values are $r=0.04, \delta=0.04, \sigma=0.2$, and $I=1$.

results also hold for the benchmark model where evaluation costs are zero (Dixit and Pindyck, 1994, p. 155). However, as evaluation costs rise, $\delta$ becomes relatively more important than $\sigma$ in determining the investment threshold and the abandonment threshold.

Table 3 shows how long the firm should wait before reevaluating projects that are neither good enough to undertake immediately nor bad enough to abandon. The first row gives the optimal time until the project is reevaluated for three different levels of the evaluation cost and three different values of $V$. For example, if the cost of evaluating the project is one percent of its cost of construction, then the firm will wait 6.3 years before reevaluating a break-even project; even small evaluation costs can lead to long delays until reevaluation. Each cell in the bottom two rows of Table 3 shows the percentage change in the optimal time until the project is reevaluated resulting from a one percent change in the corresponding parameter.

Firstly, consider the relationship between $\tau^{e}(V)$ and $V$ for a given $C$. Projects that are more valuable, but still not valuable enough to be undertaken immediately, will be reevaluated sooner than less valuable projects. The intuition is straightforward. When $V$ is high, the next evaluation is more likely to lead to investment than abandonment, in which case the firm wants the decision to occur soon $\left(\tau^{e}\right.$ is small) in order to raise the present value of the investment payoff. On the other hand, if $V$ is low, the next evaluation is more likely to lead to abandonment, in which case the firm wants the decision to occur late ( $\tau^{e}$ is large) in order to reduce the present value of the evaluation cost. Secondly, consider the relationship between $\tau^{e}(V)$ and $C$ for a 
Table 3: Optimal timing of project evaluation

\begin{tabular}{cccccccccc}
\hline \hline & \multicolumn{3}{c}{$C=0.01$} & \multicolumn{3}{c}{$C=0.02$} & \multicolumn{3}{c}{$C=0.05$} \\
& $V=0.8$ & $V=1.0$ & $V=1.2$ & $V=0.8$ & $V=1.0$ & $V=1.2$ & $V=0.8$ & $V=1.0$ & $V=1.2$ \\
\hline$\tau^{e}(V)$ & 9.2 & 6.3 & 4.1 & 11.0 & 7.4 & 4.7 & 15.9 & 10.5 & 6.6 \\
\hline & & & \multicolumn{9}{c}{ Elasticities } \\
$\delta$ & -0.91 & -1.30 & -1.86 & -0.78 & -1.21 & -1.84 & -0.54 & -1.02 & -1.75 \\
$\sigma$ & -0.60 & -0.27 & 0.25 & -0.69 & -0.35 & 0.17 & -0.80 & -0.47 & 0.08 \\
\hline \hline
\end{tabular}

Notes. This table illustrates the sensitivity of the optimal time until the project is reevaluated $\left(\tau^{e}\right)$ to changes in parameters describing the stochastic structure of the completed project's value. The first row gives the optimal time until the project is reevaluated for three different levels of the evaluation cost and three different values of $V$. Each cell in the following two rows shows the percentage change in the optimal time until the project is reevaluated resulting from a one percent change in the corresponding parameter. The baseline parameter values are $r=0.04, \delta=0.04$, $\sigma=0.2$, and $I=1$.

given $V$. Projects with higher evaluation costs will be reevaluated later than those with lower evaluation costs because the desire to reduce the present value of the evaluation expenditure is stronger than the desire to raise the present value of the evaluation payoff. When combined with the results from Table 2, this means that firms facing higher evaluation costs will wait a longer time before reevaluating a project, but will then be more likely to make a final decision to undertake or abandon a project; in short, evaluations will be few and far between.

The second row of Table 3 shows that reevaluations occur sooner when $\delta$ is large. As discussed earlier, a higher value of $\delta$ raises the opportunity cost of waiting and reduces the expected growth rate of $V_{t}$ if the firm delays investment. Both give the firm a greater incentive to invest earlier, which can only be achieved if the project is reevaluated earlier. The effect of volatility on reevaluation timing is slightly more complicated, as can be seen from the change in sign in the bottom row of Table 3. The intuition is clearest if we consider what happens when volatility falls. It is then going to take longer, on average, for the project's value to rise or fall sufficiently far for the next project evaluation to be decisive, all else equal. The firm's desire to reduce the number of costly evaluations therefore leads to a greater delay until the project is next evaluated; that is, lower $\sigma$ results in a larger $\tau^{e}$. However, Table 2 shows that the investment threshold will fall, making it more likely that the next evaluation will lead to immediate investment. The firm's desire to maximize the present value of the investment payoff leads it to reevaluate the project sooner; that is, lower $\sigma$ leads to lower $\tau^{e}$. The bottom row of Table 3 shows that this effect dominates the first one when $V$ is large (and future investment is relatively likely). ${ }^{8}$

\footnotetext{
${ }^{8}$ Table 2 also shows that the abandonment threshold will rise, making it more likely that the next evaluation
} 
Inspection of Tables 2 and 3 shows that the project's payout rate $\delta$ and volatility $\sigma$ affect the overall investment policy in quite different ways. Raising $\delta$ leads the investment threshold to fall, the abandonment threshold to rise, and projects between these thresholds to be reevaluated sooner. A high value of $\delta$ makes delaying investment expensive (due to more foregone cash flows), so that the decision-making process is accelerated - there are frequent evaluations and 'decisive' decision-making. In contrast, raising the volatility $\sigma$ leads the investment threshold to rise, the abandonment threshold to fall, and projects between these thresholds to be reevaluated sooner (unless $V$ is particularly high). A high value of $\sigma$ makes delaying investment attractive (since payoff extremes can be reached quickly), but because the high volatility means that a lot can happen in a short space of time, it is optimal to evaluate the project frequently - there are frequent evaluations, but 'indecisive' decision-making.

\subsection{Mean reversion}

Much of the value of a project's investment rights comes from having the flexibility to invest at exactly the right time, but if the project is only evaluated once a year, for example, then a valuable opportunity to invest midyear might be missed. This will be less important when project value follows geometric Brownian motion, since stochastic shocks to project value are permanent in that case, but when project value is mean reverting the cost of missing the 'peak' project value could be significant. In this section I investigate this possibility by analyzing the model of Section 2.1 when project value is mean reverting. This allows me to analyze the determinants of the value of timing flexibility; in particular, to identify what makes timing flexibility (or, rather, the ability to frequently reassess the project) so valuable.

Following Dixit and Pindyck (1994, pp. 161-167), I suppose that the process for $V_{t}$ is

$$
d V_{t}=\eta\left(M-V_{t}\right) V_{t} d t+\sigma V_{t} d \xi_{t}
$$

where $\eta$ is the constant rate of mean reversion, $M$ is the long-run level of $V_{t}$, and $\sigma$ determines volatility. ${ }^{9}$ Table 4 shows the effect of costly project evaluation on the value of the firm's investment timing option when $r=0.04, \sigma=0.2, I=1$, and $\eta$ varies between 0.10 and 0.20. The entries in the top panel of the table give the percentage reduction in the value of the project rights due purely to altered investment timing, for different combinations of $V$ and $M$, relative to the benchmark case where project evaluations are costless. In terms of equation (3), they report $P_{0}^{i}(V)-P_{C}^{i}(V)$, expressed as a percentage of $P_{0}^{i}(V)$. The entries in the bottom panel of the table give the percentage reduction in the value of the project rights due to the expenditure on project evaluations, relative to the same benchmark. In terms of equation (3), they report

will lead to immediate abandonment. The firm's desire to minimize the present value of evaluation expenditure leads it to reevaluate the project later; that is, lower $\sigma$ leads to higher $\tau^{e}$. This effect thus reinforces the first one.

${ }^{9}$ Equivalently, the completed project generates cash flow of $\delta\left(V_{t}\right)=\left(r+\eta\left(V_{t}-M\right)\right) V_{t}$ at date $t$. 
Table 4: The effect of costly project evaluation on the value of the firm's investment timing option when project value is mean reverting

\begin{tabular}{|c|c|c|c|c|c|c|c|c|c|}
\hline & \multicolumn{3}{|c|}{$C=0.01$} & \multicolumn{3}{|c|}{$C=0.02$} & \multicolumn{3}{|c|}{$C=0.05$} \\
\hline & $V=0.8$ & $V=1.0$ & $V=1.2$ & $V=0.8$ & $V=1.0$ & $V=1.2$ & $V=0.8$ & $V=1.0$ & $V=1.2$ \\
\hline & \multicolumn{9}{|c|}{ Cost of distorted investment timing } \\
\hline & \multicolumn{9}{|c|}{$\eta=0.10$} \\
\hline$M=0.8$ & 30.9 & 24.7 & 15.9 & 33.0 & 25.6 & 25.5 & 61.7 & 45.3 & 25.5 \\
\hline$M=1.0$ & 21.8 & 18.5 & 14.1 & 25.4 & 21.5 & 16.3 & 40.3 & 33.1 & 38.2 \\
\hline \multirow[t]{2}{*}{$M=1.2$} & 17.0 & 15.2 & 12.7 & 19.4 & 17.3 & 14.5 & 29.7 & 25.9 & 21.4 \\
\hline & \multicolumn{9}{|c|}{$\eta=0.15$} \\
\hline$M=0.8$ & 37.5 & 29.8 & 19.9 & 44.8 & 35.1 & 19.9 & 100.0 & 56.5 & 19.9 \\
\hline$M=1.0$ & 25.8 & 22.4 & 16.8 & 29.5 & 25.4 & 19.2 & 42.6 & 35.4 & 36.6 \\
\hline \multirow[t]{2}{*}{$M=1.2$} & 18.6 & 17.0 & 14.6 & 20.9 & 19.1 & 16.4 & 31.1 & 28.1 & 23.9 \\
\hline & \multicolumn{9}{|c|}{$\eta=0.20$} \\
\hline$M=0.8$ & 42.6 & 33.7 & 15.2 & 50.9 & 39.5 & 15.2 & 100.0 & 64.3 & 15.2 \\
\hline$M=1.0$ & 28.5 & 25.2 & 18.7 & 32.2 & 28.3 & 21.1 & 46.0 & 38.8 & 35.1 \\
\hline \multirow[t]{3}{*}{$M=1.2$} & 19.4 & 18.0 & 15.7 & 21.5 & 20.0 & 17.4 & 31.5 & 29.0 & 25.0 \\
\hline & \multicolumn{9}{|c|}{ Cost of evaluation expenditure } \\
\hline & \multicolumn{9}{|c|}{$\eta=0.10$} \\
\hline$M=0.8$ & 9.7 & 7.1 & 4.7 & 28.3 & 22.8 & 0.0 & 34.5 & 33.7 & 0.0 \\
\hline$M=1.0$ & 7.8 & 6.5 & 5.1 & 19.5 & 16.7 & 12.8 & 34.2 & 31.4 & 0.0 \\
\hline \multirow[t]{2}{*}{$M=1.2$} & 5.2 & 4.7 & 4.0 & 14.4 & 13.0 & 11.0 & 26.9 & 25.3 & 21.8 \\
\hline & \multicolumn{9}{|c|}{$\eta=0.15$} \\
\hline$M=0.8$ & 11.0 & 8.5 & 0.0 & 27.5 & 22.4 & 0.0 & 0.0 & 32.2 & 0.0 \\
\hline$M=1.0$ & 6.3 & 5.6 & 4.6 & 18.9 & 16.9 & 12.8 & 36.5 & 35.0 & 0.0 \\
\hline \multirow[t]{2}{*}{$M=1.2$} & 4.2 & 3.9 & 3.6 & 13.5 & 12.6 & 11.0 & 25.7 & 24.8 & 21.8 \\
\hline & \multicolumn{9}{|c|}{$\eta=0.20$} \\
\hline$M=0.8$ & 13.1 & 10.4 & 0.0 & 30.8 & 25.9 & 0.0 & 0.0 & 30.9 & 0.0 \\
\hline$M=1.0$ & 5.7 & 5.2 & 4.4 & 19.0 & 17.4 & 13.0 & 36.4 & 36.0 & 0.0 \\
\hline$M=1.2$ & 3.9 & 3.7 & 3.5 & 13.4 & 12.8 & 11.4 & 25.4 & 25.0 & 22.4 \\
\hline
\end{tabular}

Notes. The entries in the top panel of the table give the loss in value of the project rights that is due to the altered investment timing $\left(P_{0}^{i}(V)-P_{C}^{i}(V)\right)$, expressed as a percentage of $P_{0}^{i}(V)$, for different combinations of $V, M$, and $\eta$. The entries in the bottom panel give the loss in value that is due to the cost of carrying out the required evaluations $\left(P^{e}(V)\right)$, expressed as a percentage of $P_{0}^{i}(V)$, for different combinations of $V, M$, and $\eta$. Project value evolves according to the mean reverting process in (4). Parameter values are $r=0.04, \sigma=0.2$, and $I=1$. 
$P^{e}(V)$, expressed as a percentage of $P_{0}^{i}(V)$. For example, if $M=V=I$ and $\eta=0.20$ (so that the project will quickly revert to being a break-even proposition), then raising the evaluation cost from zero to one percent of the initial outlay lowers the value of the firm's timing option by 30.4 percent. The altered investment timing lowers the value of the project rights by 25.2 percent of the benchmark value, while the evaluation expenditure contributes a further 5.2 percent.

The first point to note from Table 4 is that the loss of option value can be severe when project value is mean reverting. ${ }^{10}$ In some cases the timing option is lost altogether. Secondly, in almost all cases, the indirect effect of distorted timing is at least as great as the direct effect of evaluation expenditure, and in most cases is significantly greater. Thirdly, the indirect impact on the value of project rights is biggest (in absolute terms, as well as relative to the direct impact) whenever precise investment timing is most important. This is the case when mean reversion is rapid and when the project's NPV is expected to be negative in the long run (that is, when $M<I)$ ). In most of the cases shown in the table, more rapid mean reversion increases the loss in value due to positive evaluation costs. This is because more rapid mean reversion means that the project will have a positive NPV for shorter periods of time, forcing the firm to monitor the project more closely, and incur the evaluation costs more often, if it is to invest when the NPV of doing so is high. The only exceptions to this rule occur for those combinations of parameters where the evaluation costs induce the firm to invest immediately - there the figures in the table are capturing the effect of greater mean reversion on the value of the project rights with costless evaluation since, in all of these cases, more rapid mean reversion lowers the value of the timing option (when $C=0$ ) and therefore appears to reduce the loss in value due to evaluation costs. Similarly, evaluation costs have a more negative impact on the value of project rights when $M$ is low than when it is high because, as we would expect, precise timing is more important when the project is expected to have a negative NPV in the long run. Again, the only exception to this occurs when immediate investment is optimal.

In summary, when project value is mean reverting, choosing exactly the right time to invest is important: if reversion to the mean is very slow, imprecise timing is less important, but if reversion is very rapid then profitable opportunities can be quickly lost. Even apparently small evaluation costs can dramatically reduce the value of the firm's investment timing options by making it expensive for the firm to precisely time its investment. The result: real options analysis may be misleading for firms in industries where project values are mean reverting and projects are costly to evaluate.

\footnotetext{
${ }^{10}$ This is also evident from comparing these results with those in Table 1, which shows that evaluation costs have a much bigger negative impact on the value of the firm's investment timing options when project value is mean reverting.
} 


\section{Optimal investment timing when some information is costless}

In reality, firms can observe some information about their projects' values at relatively low cost, so that Section 2 makes overly pessimistic assumptions about firms' access to information. For example, commodity prices, exchange rates, and interest rates can all be observed almost costlessly and, for many projects, their impact on the value of a project can be easily calculated. Therefore, in this section I extend the model of Section 2 by supposing that some information about the project's value is costlessly available, so that the firm only incurs the evaluation cost when it wants to measure the precise value of the completed project. In between costly evaluations the firm can use the information observed costlessly, which I call generic information, to calculate an estimate of project value. The availability of costless information fundamentally alters the firm's optimal project evaluation policy — instead of reevaluating the project after a specified number of years, as in the previous section, the optimal evaluation policy is now to reevaluate the project as soon as estimated project value exceeds a specified level. ${ }^{11}$ This threshold depends on the length of time elapsed since the last evaluation. ${ }^{12}$

As in Section 2.2, I suppose that the value of the completed project evolves according to geometric Brownian motion

$$
d V_{t}=\mu V_{t} d t+\sigma V_{t} d \xi_{t},
$$

where the precise value of $V_{t}$ can only be observed if the firm incurs the evaluation cost $C$. Like Merton (1998), I assume that there is an arbitrary number of risky assets, together with a riskless asset, which trade continuously and whose prices follow correlated geometric Brownian motions. Merton shows that the value $S_{t}$ of the portfolio of continuously traded assets which has the smallest "tracking error" in mimicking $V_{t}$ evolves according to

$$
d S_{t}=\alpha S_{t} d t+\rho \sigma S_{t} d \zeta_{t}
$$

where $\alpha$ is a constant and $\zeta_{t}$ is a Wiener process with $\left(d \xi_{t}\right)\left(d \zeta_{t}\right)=\rho d t$ for some $\rho \in[0,1] .{ }^{13} S_{t}$ can be observed by the firm without cost. The 'error term' $X_{t}=V_{t} / S_{t}$ evolves according to

$$
d X_{t}=(\mu-\alpha) X_{t} d t+\left(1-\rho^{2}\right)^{1 / 2} \sigma X_{t} d \eta_{t}
$$

where $d \eta_{t}=\left(d \xi_{t}-\rho d \zeta_{t}\right) /\left(1-\rho^{2}\right)^{1 / 2}$ and $d \zeta_{t}$ are uncorrelated. In fact, $d X_{t}$ is uncorrelated with the returns of all traded assets. Like Merton, I therefore assume that the risk of changes in this

\footnotetext{
${ }^{11}$ In practice, this might take the form of reevaluating the project as soon as interest rates fall below a particular level or commodity prices rise above a particular level.

${ }^{12}$ Epstein et al. (1999) and Childs et al. (2001) consider a firm with a perpetual option to invest in a project of unknown value. In both models the firm can observe a noisy signal of the project's value while it waits to invest, but cannot learn the true value of the project prior to investing. Epstein et al. (1999) assume that the value of the underlying asset is constant, so that uncertainty falls as the firm waits. In contrast, Childs et al. (2001) allow the project's value to evolve over time so that uncertainty rises over time.

${ }^{13}$ This portfolio minimizes the instantaneous variance of $d V_{t} / V_{t}-d S_{t} / S_{t}$.
} 
error term are not priced by the market. It follows that the expected rate of return from holding the completed project must equal the expected rate of return, $\alpha$, on the tracking portfolio. That is, the completed project must generate cash flow of $\delta V_{t}$ at date $t$, where $\delta=\alpha-\mu$. If $\rho=0$ then traded assets provide the firm with no useful information between project evaluations; $S_{t}$ is riskless, so that $\alpha$ must equal $r$, and the growth rate of $V_{t}$ is $\mu=r-\delta$ as in Section 2.2. If $\rho=1$ then the firm can effectively observe $V_{t}$ and the results of Dixit and Pindyck (1994, Chapter 5) apply.

Rather than present the results in terms of $S_{t}$, it is more natural to present them in terms of the firm's forecast of the completed project's value: $\hat{V}_{t}=E_{t}\left[V_{t}\right]=S_{t} E_{t}\left[X_{t}\right]$. If the project was most recently evaluated at date $T$, then $\log X_{t}$ is normally distributed with mean $\log \left(V_{T} / S_{T}\right)-$ $\left(\delta+\frac{1}{2}\left(1-\rho^{2}\right) \sigma^{2}\right)(t-T)$ and variance $\left(1-\rho^{2}\right) \sigma^{2}(t-T)$. It follows that ${ }^{14}$

$$
\hat{V}_{t}=V_{T}\left(\frac{S_{t}}{S_{T}}\right) e^{-\delta(t-T)} .
$$

From Itô's Lemma, $\hat{V}_{t}$ evolves according to the geometric Brownian motion

$$
d \hat{V}_{t}=\mu \hat{V}_{t} d t+\rho \sigma \hat{V}_{t} d \zeta_{t}
$$

between project evaluations. Evaluating the project introduces a jump into the expected payoff because then $\hat{V}_{t}$ is reset to equal $V_{t}$, and the variance of this jump grows with the time elapsed since the most recent project evaluation.

The main difference from the model in Section 2 is that now the firm can use the information contained in $S_{t}$ to value the project rights and to determine its evaluation and investment policies. I show in Appendix B that the value of the project rights at date $t$ equals $F\left(\hat{V}_{t}, t-T\right)$ for some function $F$, where $T$ is the date of the most recent project evaluation. The firm has the same options as in Section 2. That is, the firm must do one of the following at date $t$ : (i) abandon the project altogether, and receive a payoff of 0; (ii) invest immediately, and receive an expected payoff (measured immediately prior to investment) of $\hat{V}_{t}-I$; (iii) reevaluate the project, and receive an expected payoff of $E_{t}\left[F\left(V_{t}, 0\right)\right]-C$; or (iv) do nothing for the next interval of length $d t$. I show in Appendix B that $F$ satisfies the system of inequalities ${ }^{15}$

$$
\begin{aligned}
F(\hat{V}, \tau) & \geq 0 \\
F(\hat{V}, \tau) & \geq \hat{V}-I, \\
F(\hat{V}, \tau) & \geq E\left[F\left(\hat{V} e^{-\frac{1}{2}\left(1-\rho^{2}\right) \sigma^{2} \tau+\left(1-\rho^{2}\right)^{1 / 2} \sigma \tau^{1 / 2} \tilde{Z}}, 0\right)\right]-C, \quad \tilde{Z} \sim N(0,1), \\
0 & \geq \frac{\partial F}{\partial t}+\frac{1}{2} \rho^{2} \sigma^{2} \hat{V}^{2} \frac{\partial^{2} F}{\partial \hat{V}^{2}}+(r-\delta) \hat{V} \frac{\partial F}{\partial \hat{V}}-r F,
\end{aligned}
$$

\footnotetext{
${ }^{14}$ Viewed from date $t, \log V_{t}$ is normally distributed with mean $\log \hat{V}_{t}-\frac{1}{2}\left(1-\rho^{2}\right) \sigma^{2}(t-T)$ and variance $\left(1-\rho^{2}\right) \sigma^{2}(t-T)$.

${ }^{15}$ The third inequality corresponds to the possible payoff of $E_{t}\left[F\left(V_{t}, 0\right)\right]-C$. It cannot simply be set equal to $F\left(\hat{V}_{t}, 0\right)-C$ because of the potential nonlinearity of $F(\cdot, 0)$.
} 
Figure 2: Optimal investment timing when some information is costless

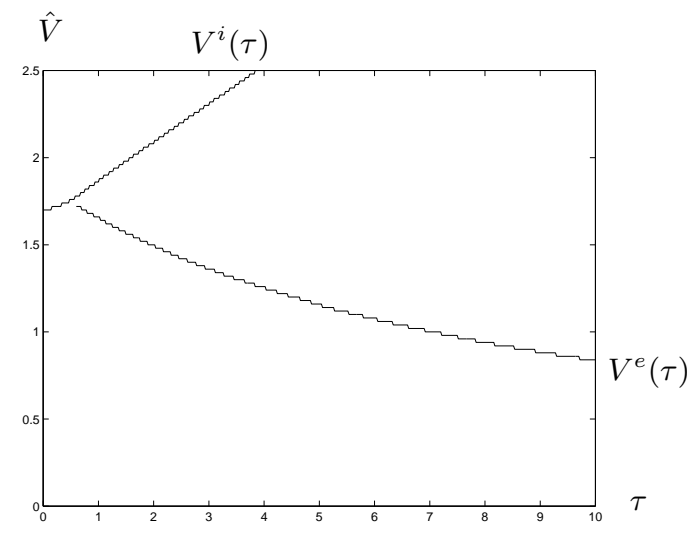

Notes. The top curve plots the investment threshold $V^{i}(\tau)$ as a function of the time $\tau$ since the project was last evaluated, while the bottom curve plots the evaluation threshold $V^{e}(\tau)$. If $\tau$ years have elapsed since the last evaluation, the firm will invest in the project if, based on information which is costlessly available, its expected value $\hat{V}$ of the completed project exceeds the threshold $V^{i}(\tau)$; it will evaluate the project if it believe the value lies between $V^{e}(\tau)$ and $V^{i}(\tau)$, otherwise it will wait. The parameters used are $r=0.04, \delta=0.04, \sigma=0.2, \rho=0.5, I=1$, and $C=0.01$.

with at least one of these restrictions holding with equality at each point. A numerical procedure for solving this problem is outlined in Appendix C.

In my numerical analysis, I find that the firm optimally chooses two thresholds, $V^{e}$ and $V^{i}$, which are both functions of the time elapsed since the project was last evaluated. If the most recent evaluation occurred on date $T$, then the firm will invest in the project at date $t$ if $\hat{V}_{t} \geq V^{i}(t-T)$, evaluate the project if $V^{e}(t-T) \leq \hat{V}_{t}<V^{i}(t-T)$, and will wait otherwise. Figure 2 plots these two thresholds for the case where $r=0.04, \delta=0.04, \sigma=0.2, \rho=0.5$, $I=1$, and $C=0.01$. It shows that immediately after an evaluation the firm will invest if $V_{t}$ exceeds 1.72 and otherwise wait. Thus, there is value in waiting. The firm will not reevaluate the project within approximately six months of a previous evaluation under any circumstances - if generic information provides sufficiently good news during this period, the firm will simply invest without any further evaluation. However, beyond this period, the firm will always evaluate the project before investment. Such evaluations will occur as soon as the project's estimated value (where the estimate is based on the project's observed value at the time of the most recent evaluation and information acquired costlessly since then) exceeds some threshold. As the time since the last evaluation grows, the standard required for reevaluation falls; if a sufficiently long time has elapsed, the firm will even reevaluate a project which it expects has a negative NPV.

The informational friction present in this model has two dimensions - the proportion of information which is project-specific (represented by 1 minus the correlation coefficient $\rho$ ), and the cost of observing this project-specific information $(C)$. The impact of these two parameters 
on the results of the numerical analysis is examined in Table 5. In all cases, $r=0.04, \delta=0.04$, and $\sigma=0.2$. Each panel of the table corresponds to a different evaluation cost. The first row of each panel gives the percentage of the value of project rights lost relative to the case where all information about the project's value can be observed without cost (that is, $C=0$ and $\rho=1$ ), for a break-even project which has just been evaluated. As might be expected, the value of the firm's timing option is lower when more information is project-specific and when project evaluations are more costly. However, the amount of project-specific information and the cost of project evaluations have subtly different effects on the firm's optimal behavior, as we now explain.

The second row gives the investment threshold $V^{i}(0)$ for a project which has just been evaluated. This threshold always exceeds the investment outlay $I$, so that investment is delayed past the break-even point, but is less than the optimal investment threshold when the project's value can be observed without cost (which, for the parameters used here, is $V^{*}=2$ ). The premium over the investment outlay falls as (i) more information is project-specific (that is, as $\rho$ falls), and (ii) project evaluation becomes more costly (that is, as $C$ rises). That is, the firm chooses a lower investment threshold when either friction becomes greater.

The remaining rows of each panel highlight the effect of informational frictions on the firm's optimal project evaluation policy. Rows 3-5 of each panel give the threshold for project evaluation, $V^{e}(\tau)$, for three different values of $\tau .{ }^{16}$ This is an increasing function of both $\rho$ and $C$, indicating that when less information is project-specific, or the cost of evaluating the project is high, the firm will wait for particularly encouraging generic information before evaluating the project. That is, the informational frictions have opposite effects on the firm's evaluation policy. In the first case, this is because the minor role of project-specific information allows the firm to perform costly project evaluations less frequently (by setting a more demanding evaluation threshold); in the second case, it is because the greater costs makes the firm reluctant to evaluate the project. ${ }^{17}$

These different effects can be understood in terms of the firm's two timing options. Firstly, the firm must decide when to evaluate the project, which it will only do once the payoff from project evaluation exceeds the value of waiting. A higher evaluation cost lowers the payoff, and so delays evaluation. If there is a great deal of project-specific information, then delaying evaluating the project yields a relatively small amount of generic information; thus, the value

\footnotetext{
${ }^{16}$ In the cases where $V^{e}(\tau)$ is reported as ' $\mathrm{n} / \mathrm{a}$ ', the firm will not reevaluate the project before investing. That is, $V^{e}(\tau)=V^{i}(\tau)$ in these situations.

${ }^{17}$ The bottom row of each panel reports the smallest value of $\tau$ for which $V^{e}(\tau)=I$, which can be interpreted as the length of time the firm will wait before evaluating what appears to be a break-even project. This provides another measure of how long the firm waits before reevaluating the project. The firm will wait longer when $\rho$ is higher and when it is more costly to evaluate the project.
} 
Table 5: Optimal investment timing when some information is costless

\begin{tabular}{lccccc}
\hline \hline$\rho$ & 0.10 & 0.25 & 0.5 & 0.75 & 0.90 \\
\hline \multirow{5}{c}{$C=0.01$} \\
\% of value lost at $V=I$ & 12.6 & 12.1 & 10.3 & 8.2 & 6.8 \\
$V^{i}(0)$ & 1.64 & 1.66 & 1.72 & 1.80 & 1.88 \\
$V^{e}(1)$ & 1.58 & 1.60 & 1.66 & $\mathrm{n} / \mathrm{a}$ & $\mathrm{n} / \mathrm{a}$ \\
$V^{e}(2)$ & 1.40 & 1.42 & 1.50 & 1.64 & $\mathrm{n} / \mathrm{a}$ \\
$V^{e}(5)$ & 1.04 & 1.06 & 1.16 & 1.36 & 1.62 \\
$\tau: V^{e}(\tau)=I$ & 5.64 & 5.92 & 7.20 & 12.30 & 25.90 \\
\hline & & \multicolumn{5}{c}{$C=0.02$} \\
$\%$ of value lost at $V=I$ & 19.6 & 18.6 & 15.9 & 11.7 & 9.1 \\
$V^{i}(0)$ & 1.52 & 1.56 & 1.64 & 1.76 & 1.86 \\
$V^{e}(1)$ & 1.60 & 1.62 & $\mathrm{n} / \mathrm{a}$ & $\mathrm{n} / \mathrm{a}$ & $\mathrm{n} / \mathrm{a}$ \\
$V^{e}(2)$ & 1.46 & 1.48 & 1.56 & $\mathrm{n} / \mathrm{a}$ & $\mathrm{n} / \mathrm{a}$ \\
$V^{e}(5)$ & 1.16 & 1.18 & 1.28 & 1.48 & 1.70 \\
$\tau: V^{e}(\tau)=I$ & 7.26 & 7.62 & 9.78 & 17.14 & 29.02 \\
\hline & \multicolumn{5}{c}{$C=0.05$} \\
$V^{i}$ of value lost at $V=I$ & 34.0 & 32.2 & 26.8 & 18.8 & 12.0 \\
$V^{i}(0)$ & 1.36 & 1.42 & 1.54 & 1.70 & 1.84 \\
$V^{e}(1)$ & $\mathrm{n} / \mathrm{a}$ & $\mathrm{n} / \mathrm{a}$ & $\mathrm{n} / \mathrm{a}$ & $\mathrm{n} / \mathrm{a}$ & $\mathrm{n} / \mathrm{a}$ \\
$V^{e}(2)$ & $\mathrm{n} / \mathrm{a}$ & $\mathrm{n} / \mathrm{a}$ & $\mathrm{n} / \mathrm{a}$ & $\mathrm{n} / \mathrm{a}$ & $\mathrm{n} / \mathrm{a}$ \\
$V^{e}(5)$ & 1.30 & 1.34 & 1.44 & $\mathrm{n} / \mathrm{a}$ & $\mathrm{n} / \mathrm{a}$ \\
$\tau: V^{e}(\tau)=I$ & 10.72 & 11.50 & 15.38 & 25.00 & 29.98 \\
\hline \hline
\end{tabular}

Notes. The first row of each panel gives the percentage of the value of project rights lost relative to the case where $C=0$ and $\rho=1$, for a break-even project which has just been evaluated. The second row gives the investment threshold $V^{i}(0)$ for a project which has just been evaluated. Rows 3-5 give the evaluation threshold $V^{e}(\tau)$ for three different values of $\tau$, and the final row gives the time which must elapse before a break-even project should be reevaluated. In the cases where $V^{e}(\tau)$ is reported as ' $\mathrm{n} / \mathrm{a}$ ', the firm will not reevaluate the project before investing; that is, $V^{e}(\tau)=V^{i}(\tau)$ in these situations. In all cases $r=0.04, \delta=0.04, \sigma=0.2$, and $I=1$. 
of waiting is low, and, all else equal, evaluation occurs sooner. ${ }^{18}$ Secondly, the firm must decide whether to launch the project or wait and reevaluate it in the future; it will only launch the project if the payoff from doing so exceeds the value of waiting. A higher evaluation cost lowers the value of waiting (by making future evaluations more expensive), and therefore promotes immediate investment. More project-specific information means that delaying yields a relatively small amount of generic information, lowering the value of waiting, and therefore also promoting immediate investment.

\section{Concluding remarks}

As it is usually applied, real options analysis assumes that projects are continuously evaluated, and launched at precisely the time determined to be optimal. However, real world projects cannot be managed in this way. The costs of formally evaluating an investment opportunity means that if it is not undertaken immediately, a project will be put to one side, to be reevaluated at some future date. Perhaps the project is subject to a 'watching brief', meaning that it is only formally evaluated again once easily observable information suggests that reevaluation is worthwhile.

This paper analyzes how projects should be managed in a (realistic) world where project evaluation is costly, extending a standard real option model by supposing that information about a project comes in two flavors - project-specific information, which can only be observed if a fixed evaluation cost is incurred, and generic information which can be observed without cost. In the first model I present, in which all information is project-specific, even small evaluation costs can significantly reduce the value of a firm's timing options. As evaluation costs grow (relative to the project's construction cost), firms should set lower investment thresholds and higher abandonment thresholds than the standard costless-evaluation analysis would suggest. If, despite this tightening of the thresholds, a firm decides to delay the investment decision, then it should wait longer when the evaluation cost is high. These distortions are greater when projects are complicated, or otherwise costly to evaluate, and when the value of the completed project is strongly mean reverting.

In the more general model, in which some (generic) information about the project's value is costlessly available, the firm only incurs the evaluation cost when it wants to measure the precise value of the completed project. If sufficiently good generic information is observed during the period immediately after a project evaluation, the firm will simply invest without any further evaluation. However, beyond this period, the firm will always evaluate the project

\footnotetext{
${ }^{18}$ In this case $\rho$ is small and, from equation (5), the volatility of $\hat{V}_{t}$ is low. The results are therefore consistent with the observation that the exercise threshold for American call options is lower when the volatility of the underlying state variable is lower.
} 
before investment. Such evaluations will occur as soon as the project's estimated value (based on the project's observed value at the time of the most recent evaluation and generic information observed since then) exceeds some threshold, which falls as the time since the last evaluation grows. The value of waiting is lower when more information is project-specific and when project evaluations are more costly. However, the availability of information and project evaluation costs affect the firm's optimal behavior in different ways: a higher evaluation cost means that the firm evaluates the project later (that is, sets a higher evaluation threshold) but invests sooner (that is, sets a lower investment threshold); a bigger role for project-specific information also induces the firm to set a lower investment threshold, but it actually encourages the firm to evaluate the project sooner.

It follows that practitioners should not simply apply models for pricing financial options when analyzing firms' real options. For instance, the timing options attached to investment in existing lines of business might be more valuable than those attached to otherwise-comparable projects for which managers are faced with greater information-gathering costs. This suggests that relatively high hurdle rates are appropriate for investment in existing lines of business, while lower hurdle rates are appropriate for new lines of business. Similarly, anything that introduces mean reversion into a completed project's value makes the value of the project rights more sensitive to evaluation costs. For example, the presence of competition can have this effect because entry and exit by competitors means that project value is more likely to revert to some long run level. Competition therefore makes it more important that investment opportunities are evaluated sufficiently frequently that transient opportunities can be exploited at the optimal time. The associated costs, both direct (involving expenditure on project evaluations) and indirect (involving distorted investment timing), reduce the value of the project rights below that implied by standard real options analysis.

\section{References}

Boyle, Glenn W. and Graeme Guthrie (2003). "Investment, uncertainty, and liquidity," Journal of Finance 58(5), 2143-2166.

Boyle, Glenn W. and Graeme Guthrie (2005). "Hedging the value of waiting," Journal of Banking and Finance (forthcoming).

Conlisk, John (1996). "Why bounded rationality?" Journal of Economic Literature 34(2), 669-700.

Childs, Paul D., Steven H. Ott, and Timothy J. Riddiough (2001). "Valuation and information acquisition policy for claims written on noisy real assets," Financial Management 30(2), $45-75$. 
Dixit, Avinash K. and Robert S. Pindyck (1994). Investment Under Uncertainty, Princeton: Princeton University Press.

Epstein, David, Nick Mayor, Philipp Schönbucher, Elizabeth Whalley, and Paul Wilmott (1999). "The value of market research when a firm is learning: Real options pricing and optimal filtering," in Lenos Trigeorgis (ed.), Real Options and Business Strategy: Applications to Decision Making, London: Risk Books.

Grenadier, Steven R. (2002). "Option exercise games: An application to the equilibrium investment strategies of firms," Review of Financial Studies 15, 691-721.

Grenadier, Steven R. and Neng Wang (2005). "Investment timing, agency and information," Journal of Financial Economics 75(3), 493-533.

Hugonnier, Julien and Erwan Morellec (2004). "Investment under uncertainty and incomplete markets," Working paper, University of Rochester.

Lambrecht, Bart M. and William R. M. Perraudin (2003). "Real options and preemption under incomplete information," Journal of Economic Dynamics and Control 27(4), 619-643.

Lyandres, Evgeny and Alexei Zhdanov (2003). "Underinvestment or overinvestment? The effect of debt maturity on investment," Simon Business School Working Paper No. FR 03-28. http://ssrn.com/abstract $=446681$

Martzoukos, Spiros H. and Lenos Trigeorgis (2001). "Resolving a real options paradox with incomplete information: After all, why learn?" Working paper, University of Cyprus.

Mauer, David C. and Sudipto Sarkar (forthcoming). "Real options, agency conflicts, and optimal capital structure," Journal of Banking and Finance.

McDonald, Robert L. and Daniel Siegel (1986). "The value of waiting to invest," Quarterly Journal of Economics 101, 707-727.

Merton, Robert C. (1998). "Applications of option-pricing theory: Twenty-five years later," American Economic Review 88(3), 323-349.

Metcalf, Gilbert E. and Kevin A. Hasset (1995). "Investment under alternative return assumptions: Comparing random walks and mean reversion," Journal of Economic Dynamics and Control 19, 1471-1488.

Novy-Marx, Robert (2004). "An equilibrium model of investment under uncertainty," Working paper, University of California, Berkeley. 
Sabarwal, Tarun (2003). "The non-neutrality of debt in investment timing: A new NPV rule," Annals of Finance, forthcoming.

Sarkar, Sudipto (2003). "The effect of mean reversion on investment under uncertainty," Journal of Economic Dynamics and Control 28, 377-396.

Schwartz, Eduardo S. (1997). "The stochastic behavior of commodity prices: Implications for valuation and hedging," Journal of Finance 52(3), Papers and Proceedings Fifty-Seventh Annual Meeting, American Finance Association, 923-973.

\section{Appendices}

\section{A Numerical solution of the model in Section 2}

Define

$$
G(V, \tau)=e^{-r \tau} E\left[F\left(V_{\tau}, 0\right)-C \mid V_{0}=V\right]
$$

and

$$
H(V, \tau)=e^{-r \tau} E\left[V_{\tau}-I \mid V_{0}=V\right] .
$$

$G$ and $H$ are determined by the partial differential equations

$$
\frac{\partial G}{\partial \tau}=\frac{1}{2} \sigma(V)^{2} \frac{\partial^{2} G}{\partial V^{2}}+(r V-\delta(V)) \frac{\partial G}{\partial V}-r G
$$

and

$$
\frac{\partial H}{\partial \tau}=\frac{1}{2} \sigma(V)^{2} \frac{\partial^{2} H}{\partial V^{2}}+(r V-\delta(V)) \frac{\partial H}{\partial V}-r H,
$$

together with their respective initial conditions

$$
G(V, 0)=F(V, 0)-C
$$

and

$$
H(V, 0)=V-I
$$

I look for functions $F(\cdot, 0), G$ and $H$ such that

$$
F(V, 0)=\max \left\{0, \sup _{\tau} G(V, \tau), \sup _{\tau} H(V, \tau)\right\} .
$$

The solution algorithm uses a grid with points labelled $\left(V_{k}, \tau_{n}\right)$, where $V_{k}=k d V$ for $k=$ $0,1, \ldots, K$ and $\tau_{n}=n d t$ for $n=0,1, \ldots, N$. I look for a vector of values $F_{k}$ representing $F\left(V_{k}, 0\right)$ such that the corresponding solutions for $G_{k}^{n}=G\left(V_{k}, \tau_{n}\right)$ and $H_{k}^{n}=H\left(V_{k}, \tau_{n}\right)$ satisfy

$$
F_{k}=\max \left\{0, \max _{n} G_{k}^{n}, \max _{n} H_{k}^{n}\right\} .
$$


The algorithm starts by setting $F_{k}=\max \left\{V_{k}-I, 0\right\}$, solves for $G$ and $H$ using the CrankNicholson finite difference method, and then updates the $F_{k}$ s using (A-1). I continue iterating in this way until the largest change in any $F_{k}$ is less than some threshold $\varepsilon$. In the numerical analysis reported in Section 2, $I=1$ and the tolerance has been set at $\varepsilon=0.0001$. Typically fewer than ten iterations were required to achieve convergence.

\section{B Derivation of inequalities determining $F$ in Section 3}

Suppose that, from the point of view of date $t$, the most recent project evaluation occurred at date $T$. Then at date $t$ the information known to the firm is the current value $S_{t}$ of the 'tracking portfolio' and the length of time that has elapsed since the project was last evaluated, together with the value of this portfolio and the actual value of the project at the time of the last evaluation. I therefore write the value of the project rights at date $t$ as $G\left(S_{t}, t-T, V_{T}, S_{T}\right)$ for some function $G$. The firm has the same options as in Section 2. That is, the firm must do one of the following at date $t$ : (i) abandon the project altogether, and receive a payoff of 0; (ii) invest immediately, and receive a payoff of $S_{t} X_{t}-I$; (iii) reevaluate the project, and receive a payoff of $G\left(S_{t}, 0, S_{t} X_{t}, S_{t}\right)-C$; or (iv) do nothing for the next interval of length $d t$, and receive a payoff of $G\left(S_{t+d t}, t+d t-T, V_{T}, S_{T}\right)$. Thus, the value of the project rights at date $t$ is

$$
\begin{array}{r}
G\left(S_{t}, t-T, V_{T}, S_{T}\right)=\max \left\{0, E_{t}\left[S_{t} X_{t}\right]-I, E_{t}\left[G\left(S_{t}, 0, S_{t} X_{t}, S_{t}\right)\right]-C,\right. \\
\left.e^{-r d t} E_{t}^{*}\left[G\left(S_{t+d t}, t+d t-T, V_{T}, S_{T}\right)\right]\right\},
\end{array}
$$

where $\log X_{t}$ is normally distributed with mean $\log \left(V_{T} / S_{T}\right)-\left(\delta+\frac{1}{2}\left(1-\rho^{2}\right) \sigma^{2}\right)(t-T)$ and variance $\left(1-\rho^{2}\right) \sigma^{2}(t-T)$, and the appearance of $E_{t}^{*}$ indicates that the value of waiting is calculated using the 'risk neutral' process for $S_{t}$. It follows that $G$ must satisfy

$$
\begin{aligned}
G\left(S_{t}, t-T, V_{T}, S_{T}\right) & \geq 0, \\
G\left(S_{t}, t-T, V_{T}, S_{T}\right) & \geq V_{T}\left(\frac{S_{t}}{S_{T}}\right) e^{-\delta(t-T)}-I, \\
G\left(S_{t}, t-T, V_{T}, S_{T}\right) & \geq E_{t}\left[G\left(S_{t}, 0, S_{t} X_{t}, S_{t}\right)\right]-C,
\end{aligned}
$$

together with

$$
\frac{\partial G}{\partial t}+\frac{1}{2} \rho^{2} \sigma^{2} S^{2} \frac{\partial^{2} G}{\partial S^{2}}+r S \frac{\partial G}{\partial S}-r G \leq 0,
$$

with at least one of these restrictions holding with equality.

We look for a solution of the form

$$
G\left(S_{t}, t-T, V_{T}, S_{T}\right)=F\left(\frac{S_{t} e^{-\delta(t-T)} V_{T}}{S_{T}}, t-T\right)
$$


and find that $F$ satisfies the system of inequalities

$$
\begin{aligned}
F(\hat{V}, \tau) & \geq 0 \\
F(\hat{V}, \tau) & \geq \hat{V}-I \\
F(\hat{V}, \tau) & \geq E\left[F\left(\hat{V} \exp \left(-\frac{1}{2}\left(1-\rho^{2}\right) \sigma^{2} \tau+\left(1-\rho^{2}\right)^{1 / 2} \sigma \tau^{1 / 2} \tilde{Z}\right), 0\right)\right]-C, \\
0 & \geq \frac{\partial F}{\partial t}+\frac{1}{2} \rho^{2} \sigma^{2} \hat{V}^{2} \frac{\partial^{2} F}{\partial \hat{V}^{2}}+(r-\delta) \hat{V} \frac{\partial F}{\partial \hat{V}}-r F
\end{aligned}
$$

where $\tilde{Z}$ is drawn from the standard normal distribution.

\section{Numerical solution of the model in Section 3}

An important part of the solution algorithm involves calculating the right hand side of condition (B-1). To see how this reduces to the problem of solving a differential equation, consider the following stochastic process for $m_{t}$ :

$$
d m_{t}=\left(1-\rho^{2}\right)^{1 / 2} \sigma m_{t} d \zeta_{t}
$$

When viewed from time $0, \log m_{\tau}$ is normally distributed with mean $\log m_{0}-\frac{1}{2}\left(1-\rho^{2}\right) \sigma^{2} \tau$ and variance $\left(1-\rho^{2}\right) \sigma^{2} \tau$. Therefore, the right hand side of (B-1) equals $H(\hat{V}, \tau)-C$, where

$$
H(\hat{V}, \tau)=E_{0}\left[F\left(m_{\tau}, 0\right) \mid m_{0}=\hat{V}\right] .
$$

$H$ satisfies the partial differential equation

$$
\frac{\partial H}{\partial \tau}=\frac{1}{2}\left(1-\rho^{2}\right) \sigma^{2} \hat{V}^{2} \frac{\partial^{2} H}{\partial \hat{V}^{2}}
$$

and the initial condition $H(\hat{V}, 0)=F(\hat{V}, 0)$.

The solution algorithm uses a grid with points labelled $\left(\hat{V}_{k}, \tau_{n}\right)$, where $\hat{V}_{k}=k d \hat{V}$ for $k=$ $0,1, \ldots, K$ and $\tau_{n}=n d t$ for $n=0,1, \ldots, N$. We look for matrices $F_{k}^{n}$ and $H_{k}^{n}$ representing $F\left(\hat{V}_{k}, \tau_{n}\right)$ and $H\left(\hat{V}_{k}, \tau_{n}\right)$ respectively, such that

$$
F_{k}^{n}=\max \left\{0, \hat{V}_{k}-I, H_{k}^{n}-C, \frac{\alpha_{k} F_{k-1}^{n}+\gamma_{k} F_{k+1}^{n}+\alpha_{k}^{\prime} F_{k-1}^{n+1}-\beta_{k}^{\prime} F_{k}^{n+1}+\gamma_{k}^{\prime} F_{k+1}^{n+1}}{\beta_{k}}\right\},
$$

where

$$
\alpha_{k} F_{k-1}^{n}-\beta_{k} F_{k}^{n}+\gamma_{k} F_{k+1}^{n}+\alpha_{k}^{\prime} F_{k-1}^{n+1}-\beta_{k}^{\prime} F_{k}^{n+1}+\gamma_{k}^{\prime} F_{k+1}^{n+1}
$$

is the Crank-Nicholson approximation to

$$
\frac{\partial F}{\partial t}+\frac{1}{2} \rho^{2} \sigma^{2} \hat{V}^{2} \frac{\partial^{2} F}{\partial \hat{V}^{2}}+(r-\delta) \hat{V} \frac{\partial F}{\partial \hat{V}}-r F
$$

at $\left(\hat{V}_{k}, \tau_{n}\right)$. 
The algorithm starts by setting $F_{k}^{0}=\max \left\{V_{k}-I, 0\right\}$ and then solving for $H$ using the Crank-Nicholson finite difference method, starting with $n=0$ and solving forward until $n=N$. It then solves for $F$, starting at $n=N$ by setting ${ }^{19}$

$$
F_{k}^{N}=\max \left\{0, H_{k}^{N}-C, V_{k}-I\right\}
$$

The algorithm then works backwards, solving (C-1) at each step using projected successive overrelaxation until $n=0$. This gives new values of $F_{k}^{0}$. I then repeat the process, continuing to iterate in this way until the largest change in any $F_{k}^{0}$ is less than some threshold $\varepsilon$. In the numerical analysis reported in Section $3, I=1$ and the tolerance has been set at $\varepsilon=0.0001$. Typically fewer than 15 iterations were required to achieve convergence.

\footnotetext{
${ }^{19}$ That is, I assume that $N$ is sufficiently large that the firm will optimally do one of abandon, invest, or reevaluate if $N d t$ years have elapsed since the most recent evaluation.
} 